\title{
EVALUACIÓN IN VITRO DE HONGOS NEMATÓFAGOS EN ZONAS ARROCERAS DE COSTA RICA CONTRA EL NEMATODO AGALLADOR Meloidogyne javanica ${ }^{1}$
}

\author{
Walter Peraza Padilla ${ }^{2 *}$, Martha Orozco Aceves**, Alejandro Esquivel Hernández** \\ Palabras clave: Pruebas in vitro; hongos nematófagos; capacidad depredadora; Meloidogyne sp. \\ Keywords: In vitro test; nematophagous fungi; predatory capacity; Meloidogyne sp.
}

\begin{abstract}
RESUMEN
Se evaluó el parasitismo in vitro de 14 aislamientos de hongos nematófagos (6 Trichoderma sp., 4 Paecilomyces sp., 2 Fusarium oxysporum, y 2 Monacrosporium sp.) contra juveniles y huevos del nematodo agallador Meloidogyne javanica. Los hongos, de 3 regiones productoras de arroz en Costa Rica, (Pacífico Central, Huetar Atlántica y Chorotega), se aislaron mediante el método de espolvoreado en placas, durante abril del 2008 a agosto de 2009. Se utilizó un diseño completamente al azar con 10 repeticiones por hongo, en agar-agua (AA). Se inoculó una suspensión de 1 a $1,5 \times 10^{6}$ conidios $\left(\right.$ ufc. $\mathrm{ml}^{-1}$ ) por plato Petri, y al cuarto día se depositó en $0,5 \mathrm{ml}$ una suspensión de 150 juveniles y 100 huevos de $M$. javanica previamente desinfectados, a temperatura ambiente $\left(23-26^{\circ} \mathrm{C}\right)$ y con luz natural. Transcurridas $96 \mathrm{~h}$ se contó en cada plato el número de nematodos vivos, muertos ó parasitados y de huevos parasitados o no parasitados. Se realizó un análisis de regresión logística (RL) para las variables evaluadas, el cual mostró diferencias significativas $(p<0,0001)$ en el desempeño de los hongos evaluados, presentando rangos de entre $10 \%$ a $56 \%$ en nematodos muertos, de

1 Resultados de la tesis de Maestría en Agricultura Alternativa con Mención en Agricultura Ecológica.

2 Autor para correspondencia. Correo electrónico: walter.peraza.padilla@una.cr
\end{abstract}

\begin{abstract}
In vitro evaluation of nematophagous fungi of Costa Rica rice-growing regions against the root-knot Meloidogyne javanica. Parasitism in vitro of 14 isolates of nematophagous fungi (6 Trichoderma sp., 4 Paecilomyces sp., 2 Fusarium oxysporum and 2 Monacrosporium sp.) was evaluated againts juveniles and eggs of the root-knot nematode, Meloidogyne javanica. The fungi, from three rice-producing regions of Costa Rica (Central Pacific, Atlantic Huetar and Chorotega), were isolated by the sprinkling technique, during April 2008 to August 2009. A completely randomized design with 10 replicates in water agar (WA) per fungus was used. A suspension of 1 to $1.5 \times 10^{6}$ conidia $\left(\mathrm{cfu} . \mathrm{ml}^{-1}\right)$ per Petri dish was inoculated, and during the fourth day was added a suspension of $0.5 \mathrm{ml}$ with 150 juvenile and 100 eggs of $M$. javanica previously disinfected, at room temperature $\left(23-26^{\circ} \mathrm{C}\right)$ and with natural light. After 96 hours, it was counted in every dish the number of living, dead and parasitized nematodes, plus eggs parasitized or not parasitized. Subsequently, a logistic regression analysis (RL) for the evaluated variables was performed, which showed significant differences

\footnotetext{
* Laboratorio de Nematología, Escuela de Ciencias Agrarias. Universidad Nacional. Heredia, Costa Rica. ** Laboratorio de Microorganismos de Suelo y Control Biológico. Universidad Nacional. Heredia, Costa Rica.
} 
$13 \%$ a $79 \%$ en nematodos parasitados y de $1 \%$ a 96\% en huevos parasitados. En general, los hongos del género Trichoderma (Tri1, Tri2, Tri3 y Tri4) fueron los más eficientes en el parasitismo sobre juveniles y huevos de M. javanica.

\section{INTRODUCCIÓN}

El arroz (Oryza sativa L.) es uno de los granos de mayor importancia a nivel mundial no solo por su consumo sino por la cantidad de personas que dependen de él, sin embargo, su rendimiento se ve mermado por una serie de enfermedades y plagas entre ellas los nematodos fitoparásitos. Un género particular es el nematodo agallador Meloidogyne sp., endoparásito sedentario presente en muchos cultivos agrícolas (Casas y Herrera 2007) especialmente en regiones subtropicales y tropicales (Netscher y Sikora 1990). Entre los nematodos fitoparásitos es el más importante desde el punto de vista económico por los daños que produce (Quereshi et ál. 1984) con un ámbito de hospederos de más de 2000 especies de plantas (Barker 1985). De acuerdo con Sasser y Freckman (1987), Meloidogyne spp., es el más dañino entre los géneros de fitonematodos. En plantaciones de arroz Meloidogyne sp., puede causar pérdidas de hasta un $20 \%$ en arroz inundado y de un 30\% en arroz semi-inundado (Tandigan et ál. 1996, Prasad et ál. 1985).

Hasta el momento los nematicidas sintéticos son la principal estrategia de combate contra este patógeno, seguido de la rotación de cultivos y la utilización de plantas resistentes (Choleva et ál. 2006). No obstante, muchos de estos productos químicos son retirados del mercado por razones toxicológicas o están previstos para su eliminación gradual por lo que la investigación de opciones de control es un tema de mucha importancia $(\mathrm{p}<0.0001)$ in the performance of the evaluated isolates, presenting ranges from $10 \%$ to $56 \%$ in dead nematodes, $13 \%$ to $79 \%$ in parasitic nematodes and $1 \%$ to $96 \%$ in parasitized eggs. In general, isolates of Trichoderma sp. (Tri1, Tri2, Tri3 and Tri4) were the most efficient in the parasitism over juveniles and eggs of M. javanica.

en los últimos años (Nico et ál. 2004). En este sentido, el combate biológico con hongos antagonistas solos o en combinación con otros métodos es una alternativa más en el manejo de plagas (Agnenin 2011, Khan et ál. 2011, Soto-Barrientos et ál. 2011).

Un grupo que actualmente se estudia entre los organismos antagónicos de nematodos son los hongos nematófagos, los cuales incluyen a más de 200 especies descritas alrededor del mundo con alto potencial como controladores biológicos (Kerry 2000). De acuerdo con su estrategia de captura, estos hongos se dividen en predadores o atrapa nematodos porque producen un sistema hifal que sujeta, mata y consume a su presa (Barrón 1977), endoparásitos (Gray 1987), parásitos de huevos y hembras (Cave 1995) y productores de toxinas (Li et ál. 2000).

En Costa Rica algunos estudios evidencian que los hongos nematófagos se encuentran ampliamente distribuidos en suelos del territorio nacional, especialmente en sistemas agrícolas diversos (Orozco 2005, Barrientos et ál. 2011, Peraza et ál. 2011). Por lo tanto, el material para incorporar una estrategia de combate biológico de nematodos fitoparásitos se encuentra disponible en el país. Una vez que se cuenta con una serie de candidatos para ser usados como controladores biológicos, el siguiente paso constituye la realización de pruebas que permitan la evaluación de los mismos para seleccionar aquellos que presenten una mayor capacidad antagónica. Diversos aislamientos de hongos nematófagos 
han presentado capacidades antagónicas variables, como por ejemplo Trichoderma sp. (Eapen et ál. 2005, Santhosh et ál. 2005, Naserinasab et ál. 2011), Paecilomyces sp. (Xiujuan et ál. 2000, Siddiqui y Shaukat 2004), Fusarium sp. (Hyakumachi y Kubota 2004) y Monacrosporium sp. (González et ál. 2005). El presente estudio tiene como objetivo determinar la capacidad antagónica de hongos nematófagos aislados de zonas arroceras de Costa Rica contra huevos y juveniles del nematodo agallador $M$. javanica por medio de pruebas in vitro.

\section{MATERIALES Y MÉTODOS}

Durante abril del 2008 a enero del 2009 se realizaron 13 giras de campo en 3 regiones productoras de arroz de Costa Rica: Pacífico Central, Huetar Atlántica y Chorotega con el objetivo de aislar hongos nematófagos. Para la búsqueda de estos agentes de combate biológico se delimitaron en las fincas 3 áreas de muestreo de $4 \mathrm{~m}^{2}$ separadas entre sí por una distancia de $50 \mathrm{~m}$. En cada punto de muestreo, se recolectó con un barreno 5 submuestras de suelo a una profundidad de $20 \mathrm{~cm}$. Las muestras fueron almacenadas en bolsas de polietileno debidamente etiquetadas y trasladadas al Laboratorio de Nematología de la Escuela de Ciencias Agrarias de la Universidad Nacional en Heredia, Costa Rica, para su debido procesamiento y para realizar las pruebas de antagonismo in vitro. Con la ayuda de un GPS (Global Position System) se determinaron las coordenadas de cada punto de muestreo. Los sitios seleccionados para los muestreos presentaron las siguientes características ambientales (Cuadro 1).

Cuadro 1. Principales características ambientales de las regiones arroceras muestreadas.

\begin{tabular}{ccccccc}
\hline Región & $\begin{array}{c}\text { Precipitación } \\
(\mathrm{mm} / \text { anual })\end{array}$ & $\begin{array}{c}\text { Altitud } \\
(\mathrm{msnm})\end{array}$ & $\begin{array}{c}\text { Temp. } \\
\left({ }^{\circ} \mathrm{C}\right)\end{array}$ & $\begin{array}{c}\text { Humedad } \\
\text { Relativa } \\
(\%)\end{array}$ & $\begin{array}{c}\text { Zona de } \\
\text { vida }\end{array}$ & Cantón \\
\hline Huetar Atlántico & 3525 & 50 & 27 & 84 & Bh-T & Matina \\
& & & & & Talamanca Sarapiquí \\
\hline Pacífico Central & 3317 & 6 & 27 & 85 & Bh-T & Parrita \\
\hline Chorotega & 1200 & 20 & 28 & 70 & Bh-S & Nicoya \\
\hline
\end{tabular}

*Según clasificación de Holdridge (1982): Bh-T (Bosque Húmedo Tropical), Bh-S (Bosque Húmedo Seco).

\section{Desinfección de raíces y nematodos agalladores}

Masas de huevos y nematodos fueron obtenidas de plantaciones comerciales de tomate (Solanum lycopersicum) de la localidad de San Isidro de Heredia. Las plantas muestreadas evidenciaban síntomas visibles de agallamiento con una alta presencia de masas de huevos así como de nematodos. Para la desinfección de raíces se empleó la metodología de Barker (1985), que aunque es para extracción de huevos, permite la desinfección de los mismos ya que utiliza hipoclorito de sodio (NaOCL). Se colocó entre 20 a $50 \mathrm{~g}$ de raíz previamente lavada en un beaker con hipoclorito de sodio al $0,5 \%$ y se agitó durante 3 min. La solución se pasó a través de un tamiz de 
0,001 pulgadas (25 UM) para retener los huevos, los cuales se lavaron posteriormente con agua destilada para eliminar el exceso de cloro y permitir su desinfección superficial.

\section{Identificación de la especie de Meloidogyne mediante diseño perineal}

Hembras de Meloidogyne fueron extraídas con una aguja de disección a partir de raíces con agallas visibles. Las hembras fueron transferidas a una gota de ácido láctico al $45 \%$ y cortadas a la mitad para remover el contenido del cuerpo. En un estereoscopio y con la ayuda de un bisturí se realizaron cortes de la parte posterior con el objetivo de retirar el exceso de cutícula alrededor de la región ano-genital. Posteriormente, los diseños perineales se colocaron en un portaobjetos y se observaron en un microscopio Olympus BX50 a 100X. Cada montaje se comparó mediante la observación de los rasgos morfométricos de los patrones perineales y mediante el uso de la clave de Taylor y Sasser (1983). Se identificó taxonómicamente la especie como $M$. javanica (Treub 1885) Chitwood 1949.

\section{Preparación del inóculo y determinación de unidades formadoras de colonias (UFC)}

Catorce hongos fueron seleccionados para las pruebas in vitro (Cuadro 2) y se cultivaron en un medio papa-dextrosa-agar (PDA) de acuerdo con la metodología empleada por Khan y Goswami (2000). Esta técnica consistió en cultivar previamente sobre un medio rico en nutrientes como PDA cada uno de los hongos aislados para permitir su rápido crecimiento. Una semana después, se utilizó una asa bacteriológica cubierta con el hongo y se introdujo en un beaker con $20 \mathrm{ml}$ de agua destilada estéril con Tween 80 (surfactante y emulsificante) y se agitó por 30 segundos. Este procedimiento se realizó para cada uno de los hongos. Posteriormente homogenizada la suspensión, se realizó el conteo de esporas en un hematocímetro y se determinó la concentración de conidios (ufc. $\mathrm{ml}^{-1}$ ) a depositar por plato Petri que osciló entre 1 y $1,5 \times 10^{6}$. Pruebas preliminares de crecimiento micelial permitieron comprobar que cantidades mayores de micelio dificultaban la observación de los nematodos. Los hongos se incubaron durante 4 días a temperatura ambiente y luz natural para facilitar su crecimiento.

Cuadro 2. Géneros, especies y origen de los aislamientos utilizados en las pruebas de antagonismo in vitro contra huevos y juveniles de $M$. javanica.

\begin{tabular}{lll}
\hline Género o especie & Código & Procedencia \\
\hline Trichoderma sp. & Tri1 & Cañas, Guanacaste \\
Trichoderma sp. & Tri2 & \\
Monacrosporium sp. & Mo1 & Nicoya, Guanacaste \\
Paecilomyces sp. & Pae1 & \\
Trichoderma sp. & Tri3 & \\
Paecilomyces sp. & Pae2 & Batán, Limón \\
Monacrosporium sp. & Mo2 & \\
Trichoderma sp. & Tri4 & \\
Trichoderma sp. & Tri5 & \\
Paecilomyces sp. & Pae3 & La Delia, Sarapiquí. Heredia \\
Fusarium sp. & Fu & \\
Trichoderma sp. & Tri6 & \\
Fusarium oxysporum & FuO & Parrita, Puntarenas \\
Trichoderma sp. & Tri7 & Talamanca, Limón \\
\hline
\end{tabular}




\section{Pruebas de antagonismo in vitro}

Se establecieron repeticiones de 10 cajas Petri de $9 \mathrm{~cm}$ de diámetro por cada uno de los hongos seleccionados para evaluar el potencial antagónico. Cada caja contenía $22 \mathrm{ml}$ de AA (20 g. $\left.1^{-1}\right)$, medio pobre en nutrientes que permitió la esporulación tenue del hongo en toda el área de la placa. Seguidamente cada plato Petri se inoculó con $200 \mu \mathrm{L}$ de las suspensiones de esporas, las cuales fueron dispersadas en forma homogénea sobre la superficie del medio con un triángulo de vidrio esterilizado. Luego se incubaron de 3 a 4 días a temperatura ambiente $\left(23-26^{\circ} \mathrm{C}\right)$ y luz natural. Transcurridas $96 \mathrm{~h}$, se depositó por plato Petri $0,5 \mathrm{ml}$ de una suspensión que contenía 150 juveniles y 100 huevos de $M$. javanica estandarizados previamente mediante el conteo de 5 alícuotas de $1 \mathrm{ml}$. Pasado el tiempo de incubación cada caja se revisó en un microscopio invertido (Oympus CK30) a una magnificación de 10X y 40X para verificar la capacidad antagónica de cada hongo mediante el conteo en el número de nematodos vivos, muertos, parasitados y huevos parasitados o no parasitados. Como controles, se establecieron 5 cajas Petri con agar-agua (AA) más el hongo y 5 cajas Petri con agar-agua (AA) con juveniles y huevos del nematodo únicamente.

\section{Evaluación (Diseño experimental)}

Se utilizó un diseño completamente al azar con 10 repeticiones por tratamiento. La unidad experimental consistió en una caja Petri con medio agar agua (AA).

\section{Variables de respuesta}

1. Número de nematodos vivos

2. Número de nematodos muertos

3. Número de nematodos parasitados

4. Número de huevos sin parasitar

5. Número de huevos parasitados

Las lecturas de las variables se realizaron a los 3 y 4 días después de la inoculación de los juveniles y huevos del nematodo. En este ensayo in vitro se distinguieron 3 categorías de larvas: a) libres y vivas, aquellas que se desplazaban por la caja continuamente; b) parasitadas, aquellas en cuyo interior se encontró estructuras fúngicas como esporas e hifas; o atrapadas en anillos como en el caso de Monacrosporium c) muertas, aquellas que permanecían rígidas o en inmovilidad permanente sin contacto del hongo. En el caso de los huevos, se determinó si estaban parasitados o no parasitados. Se calculó la capacidad depredadora o parasítica (\%) tanto de juveniles como de huevos de cada caja Petri de la siguiente manera:

$\%$ nematodos parasitados $=\underline{\mathrm{N}^{\circ} \text { de nematodos parasitados }} \times 100$ Total de nematodos

$\%$ huevos parasitados $=\underline{\mathrm{N}^{\circ} \text { de huevos parasitados }} \times 100$ Total de huevos

\section{Análisis estadístico}

Las variables obtenidas fueron de naturaleza dicotómica, ya que únicamente se observaron 2 resultados posibles: nematodos vivos o muertos, nematodos parasitados o no parasitados, huevos parasitados y no parasitados, por lo que los datos fueron transformados a lógitos para un análisis de regresión logística (RL). De esta manera, se determinó cómo fue afectada la proporción de éxito, es decir, nematodos muertos o parasitados $\mathrm{y}$ huevos parasitados por los diferentes hongos.

La función lógito fue $\mathbf{y}=\log (\mathbf{p} / \mathbf{1}-\mathbf{p})$,

Donde:

$\mathrm{y}=$ valor de lógito.

$\mathrm{p}=$ probabilidad de que ocurra con éxito en $\boldsymbol{n}$ ensayos binomiales.

$\mathrm{n}=$ número de ensayos binomiales o sea número de nematodos o de huevos por caja Petri.

Se consideró que ocurrió un éxito cuando un nematodo o huevo murió o fue parasitado. Finalmente, las proporciones estimadas de mortalidad o parasitismo de cada hongo y del testigo fueron comparados entre sí mediante la prueba de Chi-cuadrado de Wald (SAS Institute Inc. 2008). 
RESULTADOS Y DISCUSIÓN

\section{Evaluación del antagonismo in vitro de hongos nematófagos}

El análisis de regresión lineal (RL) mostró diferencias significativas en la capacidad antagónica de los 14 aislamientos de hongos nematófagos contra juveniles y huevos de $M$. javanica $(\mathrm{p}<0,0001)$ para las variables de respuesta: nematodos vivos, muertos, parasitados y huevos parasitados (Cuadro 3).

Cuadro 3. Actividad parasítica y ovicida in vitro de los hongos Trichoderma, Paecilomyces, F. oxysporum y Monacrosporium contra el nematodo M. javanica.

\begin{tabular}{|c|c|c|c|c|c|c|c|c|c|c|c|}
\hline \multirow{2}{*}{$\begin{array}{l}\text { CÓDIGO } \\
\text { Mo2 }\end{array}$} & \multirow{2}{*}{$\frac{\text { HONGO }}{\text { Monacrosporium }}$} & \multicolumn{2}{|c|}{$\begin{array}{c}\% \text { Nema } \\
\text { Vivos }\end{array}$} & \multicolumn{2}{|c|}{$\begin{array}{l}\% \text { Nema } \\
\text { Muertos }\end{array}$} & \multicolumn{2}{|c|}{$\begin{array}{c}\% \text { Nema } \\
\text { Parasitados }\end{array}$} & \multicolumn{2}{|c|}{$\begin{array}{c}\% \text { Huevos sin } \\
\text { parasitar }\end{array}$} & \multicolumn{2}{|c|}{$\begin{array}{c}\% \text { Huevos } \\
\text { parasitados }\end{array}$} \\
\hline & & 39 & $\mathrm{~g}$ & 42 & $\mathrm{~b}$ & 19 & $\mathrm{j}$ & 99 & i & 1 & i \\
\hline Mo1 & Monacrosporium & 44 & $\mathrm{~h}$ & 40 & $\mathrm{bc}$ & 17 & $\mathrm{j}$ & 99 & i & 1 & I \\
\hline Pael & Paecilomyces & 35 & $\mathrm{fg}$ & 32 & d & 34 & ghi & 52 & $\mathrm{~h}$ & 48 & $\mathrm{~h}$ \\
\hline Pae2 & Paecilomyces & 18 & $\mathrm{c}$ & 39 & $\mathrm{bc}$ & 43 & e & 52 & $\mathrm{~h}$ & 48 & $\mathrm{~h}$ \\
\hline Pae3 & Paecilomyces & 29 & e & 37 & $\mathrm{c}$ & 34 & gh & 41 & $\mathrm{f}$ & 59 & $\mathrm{f}$ \\
\hline $\mathrm{Fu}$ & Fusarium & 29 & $\mathrm{e}$ & 35 & $\mathrm{~cd}$ & 36 & fg & 46 & $\mathrm{~g}$ & 54 & $\mathrm{~g}$ \\
\hline $\mathrm{FuO}$ & F. oxysporum & 21 & $\mathrm{~cd}$ & 39 & $\mathrm{bc}$ & 41 & ef & 49 & gh & 51 & gh \\
\hline Tri2 & Trichoderma & 39 & gh & 32 & d & 30 & hi & 4 & a & 96 & a \\
\hline Tri7 & Trichoderma & 24 & d & 22 & e & 54 & d & 16 & c & 84 & $\mathrm{c}$ \\
\hline Tri6 & Trichoderma & 53 & $\mathrm{i}$ & 18 & $\mathrm{f}$ & 29 & $\mathrm{i}$ & 19 & d & 81 & d \\
\hline Tri4 & Trichoderma & 13 & $\mathrm{~b}$ & 16 & $\mathrm{f}$ & 71 & $\mathrm{~b}$ & 11 & $\mathrm{~b}$ & 89 & $\mathrm{~b}$ \\
\hline Tri5 & Trichoderma & 22 & d & 13 & $g$ & 66 & $\mathrm{c}$ & 20 & d & 80 & d \\
\hline Tri3 & Trichoderma & 9 & $\mathrm{a}$ & 12 & $\mathrm{~g}$ & 79 & $\mathrm{a}$ & 26 & $\mathrm{e}$ & 74 & $\mathrm{e}$ \\
\hline Tri1 & Trichoderma & 31 & ef & 56 & $\mathrm{a}$ & 13 & k & 5 & $\mathrm{a}$ & 95 & $\mathrm{a}$ \\
\hline Testigo & & 90 & $\mathrm{j}$ & 10 & $\mathrm{~g}$ & 0 & 0 & 100 & 0 & 0 & 0 \\
\hline
\end{tabular}

Porcentajes con diferente letra $(a, b, c, d, e, f, g, h, i, j)$ en la misma columna son significativamente diferentes $(p<0,0001)$ de acuerdo con la prueba de Chi-cuadrado de Wald.

\section{Juveniles de Meloidogyne $\left(\mathrm{J}_{2}\right)$}

Los juveniles $\left(\mathrm{J}_{2}\right)$ de $M$. javanica presentes en las cajas testigo sin hongos nematófagos presentaban movimiento después de 3 días de inoculados; sin embargo, a partir del cuarto día su movimiento fue cada vez menor para finalmente morir por no tener una fuente de alimento. En el caso de las cajas que contenían hongos nematófagos, los juveniles de $M$. javanica presentaron movimiento vigoroso un día después de ser inoculados. No obstante, a los 2 días siguientes muchos juveniles se observaron inmóviles, parasitados o atrapados en redes pegajosas. Los juveniles parasitados por los diferentes hongos se encontraron en un rango que osciló entre $13 \%$ y 79\% (Cuadro 3). El aislamiento que presentó la mayor capacidad antagónica contra juveniles de $M$. javanica fue Tri3 de Trichoderma con un $79 \%$, mientras que el que presentó la menor capacidad antagónica fue Tril de Trichoderma con un $13 \%$. 
En las cajas Petri inoculadas con Tri3, Pae3 y FuO correspondientes a Trichoderma, Paecilomyces y $F$. oxysporum respectivamente, se observó la presencia de esporas adheridas a la cutícula de los juveniles que posteriormente germinaron y formaron micelio que consumió el contenido de los nematodos. Por lo tanto estos hongos nematófagos pueden considerarse endoparásitos. Por otra parte, las hongos Mo1 y Mo2 de Monacrosporium formaron redes pegajosas a las cuales se adhirieron los nematodos para ser posteriormente consumidos por el hongo, por lo tanto, estos hongos fueron considerados depredadores o atrapa-nematodos.

En las cajas Petri testigo sin hongos nematófagos, los juveniles vivos se encontraron en un $90 \%$, mientras que en las cajas inoculadas con hongos nematófagos, los juveniles vivos se encontraron en un $9 \%$ a $53 \%$ (Cuadro 3). Los juveniles muertos sin señales aparentes de que fueron parasitados se encontraron en el rango de $10 \%$ a $56 \%$ principalmente por parte de los aislamientos Tri1 (56\%), Tri2 (32\%), Pae2 (39\%) y Mo1 (40\%) (Cuadro 3). Dado que el porcentaje de juveniles muertos en presencia de los aislamientos anteriores fue significativamente $(p<0,0001)$ más alto que el de los testigos (10\%), fue probable la producción de sustancias tóxicas o antibióticas por parte de los hongos antes mencionados. La producción de estas sustancias fue previamente reportada para varias especies de hongos nematófagos, Cylindrocarpon destructans, Verticillium chlamydosporium (Chen y Chen 2003); Pochonia chlamydospora, V. lecanii, P. lilacinus, Trichoderma sp. (Eapen et ál. 2005); F. oxysporum (Athman 2006) y Trichoderma harzianum (Naserinasab et ál. 2011).

\section{Huevos}

En las cajas testigo no se observaron huevos parasitados, mientras que en las cajas inoculadas con hongos nematófagos los huevos parasitados oscilaron entre $1 \%$ y $96 \%$. Los aislamientos con mayor capacidad antagónica contra huevos de $M$. javanica fueron Tri2 (96\%) y Tri1 (95\%). Los aislamientos de
Monacrosporium (Mo1 y Mo2) no presentaron actividad antagónica contra huevos de $M$. javanica por lo que los huevos sin parasitar se observaron en un $99 \%$ (Cuadro 3).

\section{Trichoderma}

Trichoderma es un hongo que presenta mecanismos directos e indirectos de antagonismo contra nematodos. Entre los mecanismos directos se encuentran el micoparasitismo y la producción de nematotoxinas (Benítez 2004). Los mecanismos indirectos consisten en la competencia por nutrientes o espacio y la antibiosis (producción de metabolitos) (Benítez 2004). De acuerdo con las observaciones y resultados obtenidos en esta investigación, es probable que los Trichoderma sp., aislados de las zonas arroceras ejercieran efectos antagónicos directos de micoparasitismo y posible efecto nematotóxico sobre juveniles de M. javanica.

El micoparasitismo se observó como una invasión micelial agresiva que finalizó con la digestión total tanto de juveniles como de huevos en sus diferentes etapas de embriogénesis (Figura 1 A, B, C y D). El micoparasitismo en Trichoderma sugiere la existencia ya documentada de enzimas líticas como B-1,3-gluconasa, $\mathrm{N}$-acetilglucosaminidasa, quitinasa, glucanasa, celulasa y proteasa y de algunos antibióticos volátiles como 6-penthyl- $\alpha$-pyrone, ácido harciánico, ácido heptelídico, alameticina, gliotoxina, gliovirina, glisoprenina, hadacidina, malformina, masoilactona, peptaibolitos, tricholín, y viridinas (Goldman et ál. 1994). Estas enzimas líticas (quitinolíticas, proteolíticas) de diversos tipos y la acción de algunos antibióticos volátiles podrían explicar la desintegración de la cutícula de juveniles y la cubierta de huevos. Además, las nematotoxinas son compuestos químicos que pueden inactivar, inmovilizar o matar nematodos (Barrón 1977, Kerry 2000, Siddiqui y Shaukat 2004a). La presencia de nematotoxinas en las cajas Petri inoculadas con Trichoderma representó un $30 \%$ en la cantidad de juveniles inmóviles sin signos de parasitosis. El número de nematodos inmóviles presente en las cajas 

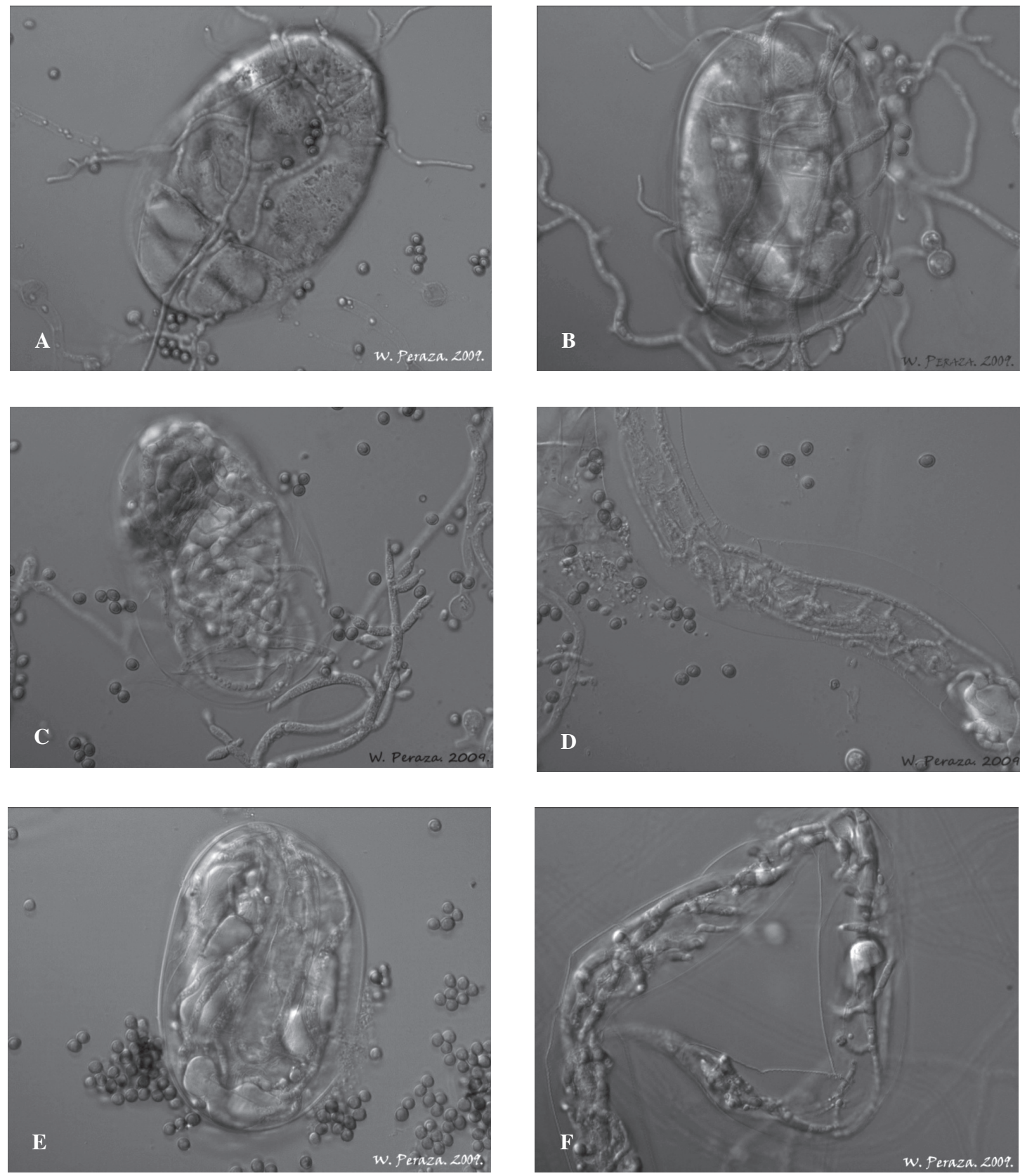

Fig. 1. (A, B, C y D). Trichoderma sp., parasitando huevos de M. javanica en varias etapas de desarrollo; el hongo consumió el contenido de los huevos y cesó el proceso de embriogénesis. (E y F) Nematodos parasitados y desintegrados por Trichoderma; se observó crecimiento micelial interno con absorción del contenido del nematodo. 100X. 
inoculadas con Trichoderma fue significativamente mayor al que se observó en los platos Petri testigos sin hongos, lo cual apoya la hipótesis de la producción y liberación al medio de nematotoxinas por parte de estos aislamientos.

Capacidades antagónicas llevadas a cabo contra Meloidogyne por parte de hongos del género Trichoderma fueron previamente reportadas por Howell (2003), González (2004), Siddiqui y Shaukat (2004a), Eapen et ál. (2005), Santhosh et ál. (2005) y Naserinasab et ál. (2011). En el caso de esta investigación, de los 7 aislamientos de Trichoderma probados, únicamente Tri3 y Tri4 presentaron una capacidad para parasitar juveniles $(>70 \%)$. Por otra parte, los 7 aislamientos de Trichoderma presentaron capacidades $(>70 \%)$ para parasitar huevos. Por lo tanto, todos los hongos del género Trichoderma demostraron un buen desempeño como micoparásitos, algunos contra juveniles y otros contra huevos de M. javanica. Desigualdades en la especificidad de los hongos nematófagos son explicadas en función de diferencias en la capa cuticular, específicamente de receptores que facilitan la interacción hongocutícula u hongo-pared al tratarse de huevos (Tunlid et ál. 1992, Jaffee y Muldoon 1995a). En general, los hongos Trichoderma aislados de zonas arroceras de Costa Rica fueron más eficientes al parasitar huevos que juveniles de $M$. javanica quizás al mostrar una actividad quitinolítica o capacidad de degradar quitina, complejo proteínico presente en la capa media de la pared de huevos. Todos los aislamientos de Trichoderma presentaron algún grado de nematoxicidad; sin embargo, Tril destacó de entre el resto de hongos debido a la presencia de más de $50 \%$ de nematodos inmóviles, por lo que podría catalogarse como el mejor productor de nematotoxinas de los 7 hongos examinados.

\section{Paecilomyces}

Al igual que Trichoderma, en el caso de Paecilomyces se identificaron como mecanismos de antagonismo contra $M$. javanica, el micoparasitismo y producción de nematotoxinas. Aislamientos de Paecilomyces tienen la capacidad de producir enzimas quitinolíticas que pueden destruir la pared de los huevos y la cutícula de juveniles (Zaki 1994, Xiujuan et ál. 2000), lo cual facilita el proceso de micoparasitismo. Por otro lado, la producción de nematotoxinas como leucinostatina y lilacinina por Paecilomyces fue previamente reportada en otras investigaciones (Barrón 1977, Kerry 2000, Xiujuan et ál. 2000, Siddiqui y Shaukat 2004). Estos datos coinciden con los de Khan et ál. (2006) al demostrar que en etapas tempranas de la embriogénesis así como en etapas más prontas a la eclosión de huevos, estos fueron parasitados por aislamientos de Paecilomyces. En los resultados obtenidos se evidenció la capacidad de infectar todas las etapas del desarrollo de huevos y juveniles de $M$. javanica. Por ejemplo, Pae1, Pae2 y Pae3 ejercieron un efecto antagónico de nematodos en un 34\%, 43\% y 34\%, mientras que parasitaron huevos en un $48 \%, 48 \%$ y $59 \%$ respectivamente. Estas diferencias eran de esperarse quizás por factores propios en la eficacia del control de estos hongos ya que pueden inhibir la capacidad de movimiento de algunos nematodos y la alteración en los procesos vitales como la eclosión y la alimentación. Como consecuencia en el caso de los nematodos afectados, pudieron morir al agotar sus reservas nutricionales y ser presa fácil de los hongos como lo menciona Marbán y Thomason (1985). De acuerdo con los resultados obtenidos, posiblemente algunas enzimas y sustancias facilitaron la capacidad antagonista debido a la mortalidad y parasitismo mostrada por estos hongos (Figura 2 A, B, C y D). 

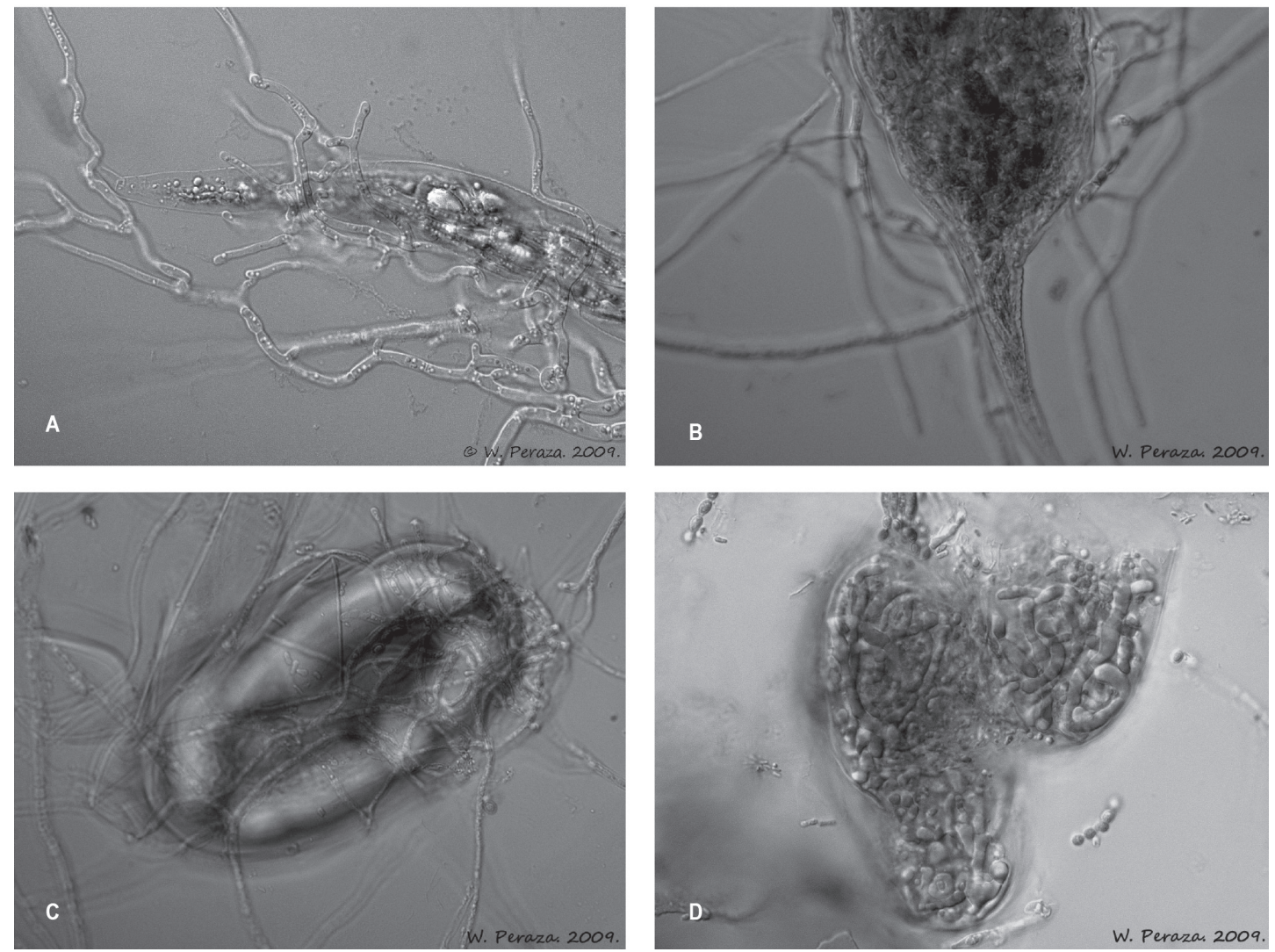

Fig. 2. (A). Huevos y juveniles de M. javanica parasitados por Paecilomyces sp. (B). Forma aberrante parasitada*. (C y D). Infección de varios estados de desarrollo de huevos de $M$. javanica. Se observó cambios morfológicos por acción ovicida. 100X. *Estado juvenil $\left(\mathrm{J}_{3} \circ \mathrm{J}_{4}\right)$ que adquiere forma de salchicha el cual deja de alimentarse y muda hasta convertirse en un adulto (macho o hembra).

\section{Fusarium}

En la naturaleza, la capacidad antagónica de Fusarium opera a través de mecanismos diferentes a los observados para Trichoderma y Paecilomyces. Algunos aislamientos de Fusarium son endófitos, los cuales colonizan raíces sin causar daño alguno a la planta hospedante. Cuando Fusarium coloniza raíces puede producir y liberar al suelo sustancias con acción repelente o nematicida (Dabatat y Sikora 2007, El-Fattah et ál. 2007). Algunos aislamientos de Fusarium en un estado endofítico pueden producir sustancias promotoras de crecimiento vegetal e inductoras de resistencia contra nematodos fitoparásitos (Hyakumachi y Kubota 2004). En ensayos in vitro, algunos hongos del género Fusarium mostraron capacidad antagónica al parasitar tanto juveniles como huevos. En esta investigación los aislamientos Fusarium sp. (Fu) y $F$. oxysporum $(\mathrm{FuO})$ mostraron una capacidad antagónica de $38,5 \%$ y $52,5 \%$ contra juveniles y huevos de $M$. javanica respectivamente (Figura 3 A y B). Datos similares fueron reportados por El-Fattah et ál. (2007) para aislamientos no patogénicos de $F$. oxysporum usados en el control de M. incognita en raíces de tomate. La presencia de juveniles inmóviles en las cajas 

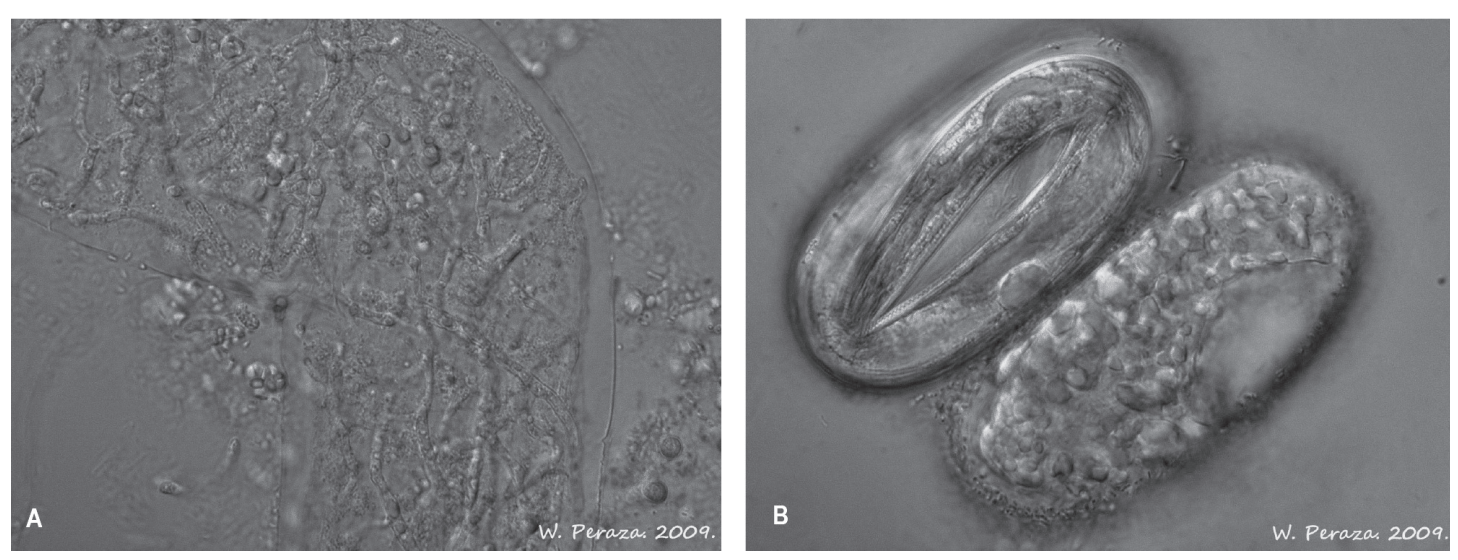

Fig. 3. (A) Forma aberrante parasitada y (B). Huevos de M. javanica. parasitados por F. oxysporum.100X.

Petri inoculadas con Fusarium sugiere la presencia de nematotoxinas en el medio, información que fue reportada previamente para este hongo por Dabatat y Sikora (2007) y El-Fattah et ál. (2007).

\section{Monacrosporium}

Por último, los aislamientos de Monacrosporium sp. (Mo1 y Mo2), mostraron un efecto parasítico bajo del $1 \%$ y $18 \%$ sobre juveniles y huevos debido quizás a que estos aislamientos presentaron una capacidad parasítica poco eficiente al comportarse más de manera saprófita que depredadora. No obstante, se observó la presencia de nematodos atrapados en redes pegajosas (Figura 4 A, B, C y D). Por otra parte, el medio de cultivo que se utilizó quizás perjudicó la efectividad del hongo en la reducción del número de estados infectivos $\left(\mathrm{J}_{2}\right)$ ya que estudios realizados por Dijksteerhuis et ál. (1994) determinaron que medios ricos en nutrientes como papa-dextrosa-agar (PDA) y harina de maíz-agar (HMA) favorecen un abundante crecimiento de micelio no así agar-agua (AA) medio que se empleó en los ensayos de esta investigación. Sin embargo, Nordbring (1998) señala que el desarrollo de micelio no está relacionado con la actividad nematófaga; no obstante, González et ál. 2005, observaron en ensayos realizados in vitro una reducción en el número de larvas cuando el crecimiento micelial fue abundante con el uso de PDA y HMA. Se comprobó que $M$. ellipsosporium controló eficientemente a $M$. incognita a nivel de invernadero y campo con reducciones en el número de agallas del 42 al 49\% (Mankau y Wu 1985). El control de $M$. javanica por acción de $M$. cionopagum en suelo fue aproximadamente del $100 \%$ en pruebas realizadas por Jaffee y Muldoon (1995b).

Esta investigación genera el primer informe de la actividad predadora in vitro de los hongos Trichoderma sp., Paecilomyces sp., F. oxysporum y Monacrosporium sp., aislados de suelos arroceros de Costa Rica contra huevos y juveniles del nematodo agallador $M$. javanica. Los hongos evaluados mostraron diferencias en la actividad antagónica, lo cual podría estar relacionado directamente con los mecanismos de acción de cada aislamiento. Con base en los antagonistas aislados, se recomienda a futuro realizar muestreos en las regiones Brunca y Huetar norte no incluidas en esta investigación. De esta manera, se podría crear una micoteca de hongos nematófagos de todas las regiones arroceras con potencial para ser utilizados como biocontroladores de nematodos. 

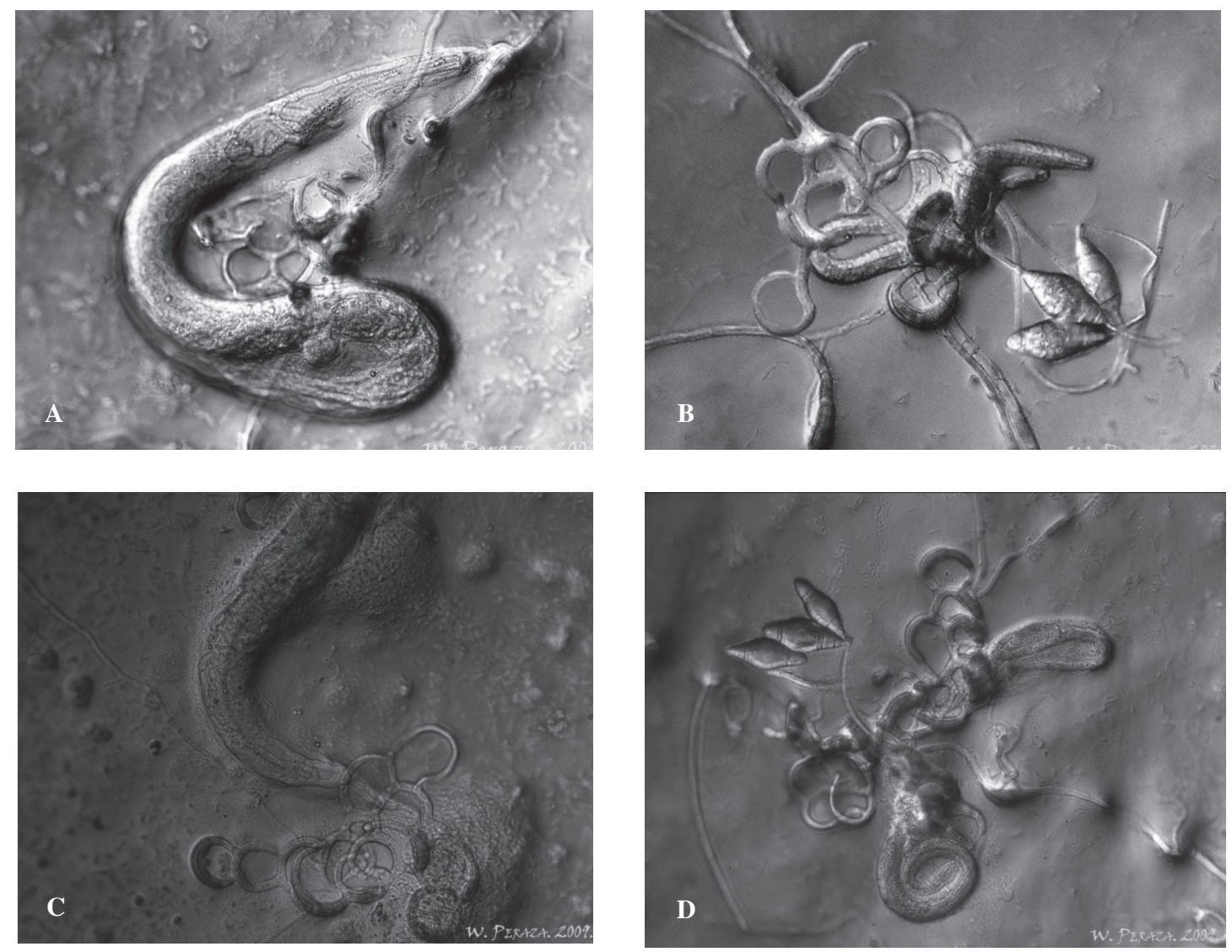

Fig. 4. A, B, C y D. M. javanica atrapado en redes pegajosas de Monacrosporium sp. (Mo1 y Mo2).

\section{AGRADECIMIENTOS}

El primer autor, da las más sentidas gracias al M.Sc. German Rivera del Laboratorio de Fitopatología, UNA; al M.Sc. Fabio Chaverri de la Escuela de Ciencias Ambientales, UNA; al Ing. Fabio Blanco, profesor pensionado de la Escuela de Ciencias Agrarias por todos sus aportes y sugerencias en la parte estadística del documento. Al "Programa interinstitucional de investigación en biodiversidad y ecología de organismos de suelo, con énfasis en sistemas de producción limpia y control biológico" financiado mediante fondos FEES (Fondos de Educación Estatal Superior), así como a la Universidad Nacional por el apoyo logístico para llevar a cabo la investigación.

\section{LITERATUA CITADA}

AGNENIN N.O. 2011. Biological control of plant parasitic nematodes: Prospects and Challenges for the Poor Africa Farmer. Plant Protection Science 47(2):62-67.

ATHMAN Y.S. 2006. Host-endophyte-pest interactions of endophytic Fusarium oxysporum antagonistic to Radopholus similis in banana (Musa spp.). $\mathrm{PhD}$ Thesis, University of Pretoria, Pretoria, South Africa. $150 \mathrm{p}$.

BARKER K.R. 1985. Nematode extracción and bioassays, pp 18-35. In: K.R. Barker, C.C. Carter and J.N. Sasser, eds. Advanced Treatise Meloidogyne. Vol. 2 Methodology. North Carolina State University, Raleigh.

BARRÓN G.L. 1977. The nematode destroying fungi. Lancaster, Pennsylvania, USA. Lancaster Press, Inc. $140 \mathrm{p}$. 
BENÍTEZ T., RINCÓN A.M., CODÓN A. 2004. Biocontrol mechanism of Trichoderma strains. International Microbiology 7:249-260.

CASAS S., HERRERA A. 2007 Antagonism of plant parasitic nematodes by fungi. The Mycota (Kubicek CP \& Druzhinina IS, eds). Springer, Berlin. p. 147157.

CAVE R. 1995. Manual para la enseñanza del control biológico en America Latina. Zamorano HN. Zamorano Academic Press. 188 p.

CHEN S.Y., CHEN, F.J. 2003. Fungal parasitism of Heterodera glycines eggs as influenced by egg age and pre-colonization of cysts by other fungi. Journal of Nematology 35:271-277.

CHITWOOD B.G. 1949. Root-knot nematodes. I. A revision of the genus Meloidogyne Goeldi, 1887. Proceedings of the Helminthological Society of Washington 16:90-104.

CHOLEVA B., BILEVA T., TZVETKOV J., VUCHKOV R. 2006. Organo-biological means and methods for control of plant parasitic nematodes as alternative of agrochemicats. In European Society of Nematologists. XXVIII International Symposium, 5-9 June, Blagoevgrad Bulgaria. $50 \mathrm{p}$.

DABABAT A.A., SIKORA R.A. 2007. Influence of the mutualistic endophyte Fusarium oxysporum 162 on Meloidogyne incognita attraction and invasion. Nematology 9(6):771-776.

DIJKSTEERHUIS J., VEENHUIS M., HARDER W., NORDBRING B. 1994. Nematophagous Fungi: Physiological aspects and structure-function relationships. Advances in Microbial Physiology (36):11-139.

EAPEN S.J., BEENA B., RAMANA K.V. 2005. Tropical soil microflora of spice-based cropping systems as potential antagonists of root-knot nematodes. Journal of Invertebrate Pathology 88:218-225.

EL-FATTAH A., DABABAT A., SIKORA R. 2007. Influence of the mutualistic endophyte Fusarium oxysporum 162 on Meloidogyne incognita attraction and invasion. Nematology 9(6):771-776.

GOLDMAN G.H., HAYES C., HARMAN G.E. 1994. Molecular and cellular biology of biocontrol by Trichoderma spp. Biotechnology Tibtech 23:10111020.

GONZÁLEZ M. 2004. Utilización de Trichoderma spp., para el control de hongos patógenos de la semilla y del suelo en el cultivo del Frijol. Fitosanidad 8(1): 61.

GONZÁLEZ R., MENDOZA P., TORRES G., BECERRIL C., ORTEGA E., HERNÁNDEZ O. 2005. Estudio in vitro de la capacidad depredadora de Duddingtonia flagrans contra larvas de nematodos gastrointestinales de ovinos de pelo. Revista Mexicana de Ciencias Pecuarias 43(3):405-414.
GRAY N.F. 1987. Nematophagous fungi with special reference to their ecology. Biological Reviews 62:245-304.

HOLDRIDGE L. 1982. Ecología basada en zona de vida. Jiménez, H. Segunda reimpresión. Instituto Interamericano de Cooperación para la Agricultura (IICA). San José, Costa Rica. 216 p.

HOWELL C.R. 2003. Mechanisms employed by Trichoderma specie sin the biological control of plant diseases: The history and evolution of current concepts plant disease 87(1):4-11.

HYAKUMACHI M., KUBOTA M. 2004. Fungi as plant growth promoter and disease suppressor, pp. 101110. In: D.K Arora (ed.). Mycology Series. Vol. 21. Fungal Biotechnology in Agricultural, Food, and Environmental Applications, Marcel Dekker, New York, NY, USA.

JAFFEE B.A., MULDOON A.E. 1995a. Susceptibility of root-knot and cyst nematodes to the nematodetrapping fungi Monacrosporium ellipsosporum and M. cionopagum. Soil Biology and Biochemistry 27:1083-1090.

JAFFEE B.A., MULDOON A.E. 1995b. Numerical responses of the nematophagous fungi Hirsutella rhossiliensis, Monacrosporium cionopagum and $M$. ellipsosporum. Mycologia 87(5):643-650.

KERRY B.R. 2000. Rhizosphere interactions and the exploitation of microbial agents for the biological control of plant-parasitic nematodes. Annual Review of Phytopathology 38:423-441.

KHAN A., WILLIAMS K.L., NEVALAINEN K.M. 2006. Control of plant-parasitic nematodes by Paecilomyces lilacinus and Monacrosporium lysipagum in pot trials. Biocontrol 51:643-658.

KHAN M.R., GOSWAMI K. 2000. Effect of culture filtrates of Paecilomyces lilacinus isolates on hatching of Meloidogyne incognita eggs. Annual Plant Protection Science 8:62-65.

KHAN T., SHADAB S., AFROZ R., ABDUL M., AZIZ A.M., FAROOQUI M. 2011. Study of Suppressive Effect of Biological agent Fungus, Natural Organic Compound and Carbofuran on Root-knot Nematode of Tomato (lycopersicon esculentum). Journal of Microbiology and Biotechnology 1(1):7-11.

LI T.F., ZHANG K.Q., LIU X.Z. 2000. Taxonomy of nematophagous fungi. Chinese Science and Technical Publishing, Beijing. 18 p.

MANKAU R., X WU. 1985. Effects of the nematode trapping fungus, Monacrosporium ellipsosporium on Meloidogyne incognita populations in field soil. Revue Nematology 8:147-153.

MARBÁN M.N., THOMASON J.I. 1985. Fitopatología avanzada I. (Eds.). Colegio de Posgraduados. Montecillo, Estado de México. MX. pp. 37-45.

NASERINASAB F., SAHEBANI N., ETEBARIAN H.R. 2011. Biological control of Meloidogyne javanica 
by Trichoderma harzianum BI and salicylic acid on tomato. African Journal of Food Science 5(4):276280.

NETSCHER C., SIKORA A.R. 1990. Nematode parasites of vegetables, pp. 237-283. In: M. Luc, A.R. Sikora and J. Bridge ed. Plant parasitic nematodes in subtropical and tropical agriculture. Wallingford, UK. CAB International.

NICO P., FENDORF S., LOWNEY Y.W., HOLM S.E., RUBY M.V. 2004. Chemical structure of arsenic and chromium in CCA-treated wood: implications of environmental weathering. Environmental Science and Technology 38(19):5253-5260.

NORDBRING B. 1998. Ecology and recognition in the nematode nematophagous fungus system. Advances in Microbial Ecology (10):81-114.

OROZCO M. 2005. Aislamiento y caracterización de hongos nematófagos como potenciales controladores biológicos de nematodos gastrointestinales para la producción animal. Tesis de maestría, Universidad Nacional, Heredia, Costa Rica. 104 p.

PERAZA W., OROZCO M., ESQUIVEL A., RIVERA G., CHAVERRI F. 2011. Aislamiento e identificación de hongos nematófagos nativos de zonas arroceraas de Costa Rica. Agronomía Mesoamerica 22(2):233-243.

PRASAD J.S., PANWAR M.S., RAO Y.S. 1985. Occurrence of root-knot nematode Meloidogyne graminicola in semi-deepwater rice. Current Science 54(8):387-388.

QUERESHI S.H., ALAM S.S., BASHIR M. 1984. Mungbean (Vigna radiate), a new host of Meloidogyne incognita in Pakistan. Pakistan Journal of Nematology 2:101-102.

SANTHOSH J., EAPEN B.B., RAMANA K.V. 2005. Tropical soil microflora of spice-based cropping systems as potential antagonists of root-knot nematodes. Journal of Invertebrate Pathology 88(3):218-225.

SASSER J.N., FRECKMAN D.W. 1987. A world perspective on nematology: the role of the society, pp. 7-14. In: J.A. Veech and D.W. Dickson (eds.). Vistas on Nematology, Society of Nematologist, Hyattsville, MD.
SAS (SAS Institute Inc. US). 2008. User's Guide. Cary, NC. $111 \mathrm{p}$.

SIDDIQUI I.A., SHAUKAT S.S. 2004. Trichoderma harzianum enhances the production of nematicidal compounds in vitro and improve biocontrol of Meloidogyne javanica by Pseudomonas fluorescens in tomato. Letters in Applied Microbiology 38:169-175.

SOTO N., DE OLIVEIRA J., VEGA R., MONTERO D., VERGAS B., HERNÁNDEZ R., OROZCO C. 2011. In-vitro predatory activity of nematophagous fungi from Costa Rica with potential use for controlling sheep and goat parasitic nematodes. Revista de Biología Tropical 59(1):37-52.

STIRLING G.R. 1991. Biological control of plant parasitic nematodes. CAB International, Wallingford. $282 \mathrm{p}$.

TANDINGAN I.C., PROT J.C., DAVIDE R.G. 1996. Influence of water management on tolerance of rice cultivars for Meloidogyne graminicola. Journal of Fundamental and Applied Nematological Research 19:189-192.

TAYLOR L.A., SASSER N.J. 1983. Biología, identificación y control de los nemátodos de nódulo de la raíz. Proyecto Internacional de Meloidogyne. Departamento de Fitopatología de la Universidad del Estado de Carolina del Norte. USA.

TREUB M. 1885. Onderzoekingen over Sereh-Ziek Suikerriet gedaan in s'lands Plantentuin te Buitenzorg. Meded. Pltuin. Batavia 2:1-39.

TUNLID A. JANSSON H.B., NORDBRING B. 1992. Fungal attachment to nematodes. Mycology Research 96:401-412.

XIUJUAN Y., XIUJUAN H., LIANG Z. 2000. Chitinasas of Paecilomyces lilacinus and studies on the biocontrol of plant parasitic nematodes. Acta Agriculturae Universitatis Jiangxiensis 22(1):86-89.

ZAKI F. 1994. Effect of culture filtrates of Paecilomyces lilacinus on Meloidogyne javanica. Nematología Mediterránea 22:41-43. 